\title{
Interaction of proteins with linear polyelectrolytes and spherical polyelectrolyte brushes in aqueous solution $\dagger$
}

\author{
A. Wittemann and M. Ballauff
}

\section{DOI: $10.1039 / \mathrm{b} 609879 \mathrm{~g}$}

We review recent experiments on the interaction of proteins with anionic polyelectrolytes in aqueous solution. Data from the literature demonstrate that proteins can form soluble complexes with linear polyelectrolytes even on the "wrong side" of the isoelectric point, that is, for $\mathrm{pH}$ values above the isoelectric point of the proteins under which the polyelectrolytes and the proteins are like-charged. All data published so far demonstrate that this type of adsorption becomes weaker with increasing ionic strength. A much stronger interaction is found if the polyelectrolyte chains are grafted onto solid surfaces to form polyelectrolyte brushes. Here it has been shown that spherical polyelectrolyte brushes consisting of a core of $c a .100 \mathrm{~nm}$ diameter and long attached polyelectrolyte chains strongly adsorb proteins at low ionic strength ("polyelectrolytemediated protein adsorption"; PMPA). Virtually no adsorption takes place onto the spherical polyelectrolyte brushes at high ionic strength. A critical comparison of data obtained on free polyelectrolytes and on polyelectrolyte brushes shows that both phenomena can be traced back to patches of positive charge on the surface of the proteins. Moreover, we discuss the driving force of the PMPA-process in terms of the Donnan pressure inside the brush layer. Here we find a good correlation which demonstrates that release of counterions during the process of adsorption is the main driving force.

\section{Introduction}

The interaction of dissolved proteins with solid surfaces is a central problem of biotechnology..$^{1-4}$ On the one hand, adsorption of proteins may be a process which must be prevented ("fouling") in technological applications. ${ }^{1,2}$ Hence, a clear understanding of the factors that lead to the attraction of proteins to solid surfaces is necessary in order to avoid this problem. On the other hand, planar and curved interfaces may serve as solid supports for the immobilization of enzymes and other biomolecules. ${ }^{1,4}$ Thus, immobilization of enzymes on suitable supports has become a central topic recently and the vast literature on this subject is hard to overlook. ${ }^{1}$ A great variety of systems designed for this purpose have been discussed recently. ${ }^{1,3,4}$ Supports suitable for technical applications must maintain a high level of enzymatic activity while preventing a possible leaching out or slow degradation during the reaction.

Central to this problem is the change of the secondary and tertiary structure of the protein molecule upon adsorption. Very often, adsorption on flat surfaces is followed by a considerable flattening and deformation of proteins. ${ }^{3,5-11} \mathrm{Si}-$ milar findings have been reported for proteins adsorbed onto the surface of colloidal particles. ${ }^{8,12}$ Hence, the surface of solid

Physikalische Chemie I, 95440 Bayreuth, Germany. E-mail: Matthias.ballauff@uni-bayreuth.de;Fax: +49 921 55 2780;Tel: + 49 921552760

$\dagger$ The HTML version of this article has been enhanced with colour images. supports must be modified in a suitable way in order to prevent a direct contact with the immobilized protein.

Surfaces to which long polymer chains have been attached may be used to prevent the adsorption of proteins from solution. ${ }^{1,2,13}$ The most important example of a protein-resistant surface coating is a dense layer of poly(ethylene oxide) (PEO) where one end of the chains is grafted to the surface. The repulsive interaction of such a layer with proteins may be explained by steric interactions: Embedding a protein within a dense layer of flexible chains leads to a loss of entropy of the system because of the reduced conformational entropy of the polymeric layer. However, even short chains of PEO may already prevent the adsorption and denaturation on solid surfaces. ${ }^{2,13}$ The repulsion between a PEO chain on the surface and the dissolved proteins must therefore be traced back to a combination of steric interaction and a repulsive interaction on the local scale. . $^{214,15}$

However, long chains of charged polymers can lead to the opposite effect, namely to strong adsorption of proteins from solution if the ionic strength in the system is low. ${ }^{16}$ If the ionic strength is high, steric repulsion prevails and the proteins do not adsorb anymore. ${ }^{16,17}$ This effect termed "polyelectrolytemediated protein adsorption" (PMPA) can be easily studied using colloidal latex particles onto which long chains of poly(acrylic acid) (PAA) or poly(styrene sulfonic acid) (PSS) have been grafted. ${ }^{18-20}$ Fig. 1 displays the structure of these particles in a schematic fashion: Long chains of either the strong polyelectrolyte PSS or the weak polyelectrolyte PAA have been grafted densely to the surface of a colloidal core of poly(styrene). The radius $R$ of the core is of the order of $50 \mathrm{~nm}$ 


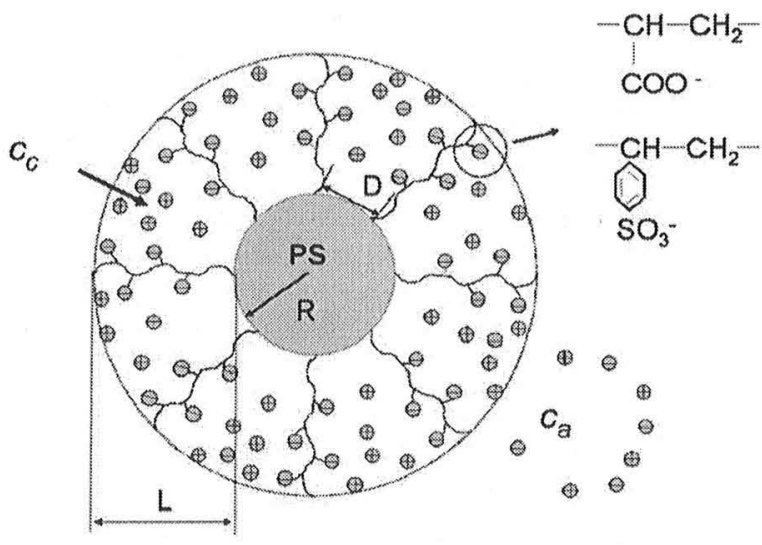

Fig. 1 Scheme of the radial structure of the spherical polyelectrolyte brushes used in previous studies of the polyelectrolyte-mediated protein adsorption. Attachment of chains of the weak polyelectrolyte poly(acrylic acid) leads to an annealed brush. ${ }^{18-22}$ If the $\mathrm{pH}$ in the system is high enough, full ionization of the charged groups within the brush layer results. ${ }^{19,20}$ If the strong polyelectrolyte poly(styrene sulfonic acid) is attached, a quenched brush results which is independent of the $\mathrm{pH}^{20}$ The average distance $D$ between the chains on the surface is of the order of a few nanometers only. Hence, the polyelectrolyte chains strongly interact and form a polyelectrolyte brush. The thickness $L$ of the brush layer can be tuned by the salt concentration $c_{a}$ in the system that determines the salt concentration $c_{c}$ within the brush layer through a Donnan equilibrium. ${ }^{16,19,20,25,32,33}$

whereas the contour length $L_{\mathrm{c}}$ of the attached chains is 10-250 $\mathrm{nm}$. The dense grafting of the polyelectrolyte chains leads to a so-called polyelectrolyte brush and the particles shown in Fig. 1 have been termed spherical polyelectrolyte brushes. ${ }^{18-22}$

The PMPA is not restricted to spherical surfaces or to colloidal systems. Czeslik et al. were able to demonstrate that this effect can be observed on planar substrates as well. , $^{37,23-25}$ By use of neutron reflectivity experiments these authors could analyze the scattering length density along the layer normal. From these data the amount of bound protein could be deduced in an accurate fashion. Moreover, the planar polyelectrolyte brushes adsorb the protein from solution only at low ionic strength. At high ionic strength there is a marked resistance against protein adsorption. Hence, the planar systems behave in the same way as the spherical systems studied earlier: There is strong adsorption at low ionic strength whereas virtually no adsorption takes place at sufficiently high ionic strength. ${ }^{25}$

Here we review recent work on the PMPA. ${ }^{16,17,23-33}$ This problem is twofold: One must first consider the interaction of proteins with linear polyelectrolyte chains in solution. ${ }^{34,35}$ In a second step the interaction of proteins with polyelectrolyte chains grafted to a surface is to be discussed. In the following we shall demonstrate that these problems are closely related to each other. Moreover, we shall present a simple model that allows us to rationalize the main experimental findings.

\section{Interaction of proteins with linear polyelectrolytes}

Proteins carry positive and negative charges on their surface and may be regarded as polyampholytes. This fact has been recognized quite early and their behaviour in solutions of electrolyte as the function of the $\mathrm{pH}$ can be understood on this basis. ${ }^{36,37}$ Depending on the $\mathrm{pH}$ proteins may have either a net positive or negative charge which is balanced at the isoelectric point. Given this fact it is obvious that proteins must interact strongly with linear polyelectrolytes of opposite charge in solution. More than 70 years ago, Bungenberg de Jong and Kruyt were the first to explore this complex formation in closer detail. ${ }^{38}$ Much of the older work has been summarized in the second volume of the treatise by Kruyt. ${ }^{39}$ Very recently, a comprehensive review was given by de Kruif and coworkers ${ }^{35}$ and by Dubin and coworkers. ${ }^{34}$

It has been recognized that proteins may interact with polyelectrolytes even on the "wrong side" of the isoelectric point $\mathrm{pI}$, that is under a $\mathrm{pH}$ where the polyelectrolytes and the proteins are like-charged. Dubin et al. (see ref. 34, 40-42 and further citations given therein) demonstrated that in this case soluble complexes may be formed at a critical value $\mathrm{pH}_{\mathrm{c}}$ above the isoelectric point. The value of $\mathrm{pH}_{\mathrm{c}}$ goes through a distinct maximum as the function of the concentration of added salt. Dubin and coworkers could explain this unexpected finding by the presence of positive "patches" on the surface of the proteins that persist beyond the isoelectric point. The interaction of linear polyelectrolytes may hence be rationalized in terms of a balance of attraction of the positive patches and the repulsion by the overall negative charge of the protein. In particular, the theory of Rubinstein and coworkers ${ }^{43}$ treating the protein as a dipole may be applied to this problem. Dubin et al. were able to show that the theory of Rubinstein et al. can give a semi-quantitative description of the strength of interaction between the polyelectrolyte and a number of proteins as e.g. bovine serum albumin (BSA). ${ }^{41}$ For an overview on this work the reader is deferred to the exhaustive review by Dubin and coworkers given in ref. 34.

A different theoretical approach for the interaction of proteins with polyelectrolytes was given by de Vries et al. ${ }^{44}$ Here the problem of complex formation is addressed in terms of polyelectrolyte adsorption on randomly charged surfaces. The strength of interaction is predicted to vary as a function of the $\mathrm{pH}$ and the concentration of added salt. Moreover, soluble complexes are expected to form at the "wrong side" if the ionic strength is low enough. de Vries et al. could correlate their experimental data with this model in a semi-quantitative fashion. More details on this approach may be found in the recent review by de Vries and coworkers. ${ }^{35}$

Concluding this brief survey, it can be stated that proteins may form weak complexes with polyelectrolytes even on the "wrong side" of the isoelectric point. This unexpected finding can be explained by the presence of positive patches on the surface of the protein which persist far above the isoelectric point. In what is to follow, it will become apparent that the PMPA is related to this finding: the proteins can now interact with the polyelectrolyte chains within a brush layer much stronger. Concomitantly, a more marked adsorption is expected.

\section{Interaction with polyelectrolyte brushes}

As mentioned above, we have demonstrated that BSA and several other proteins adsorb strongly on spherical 


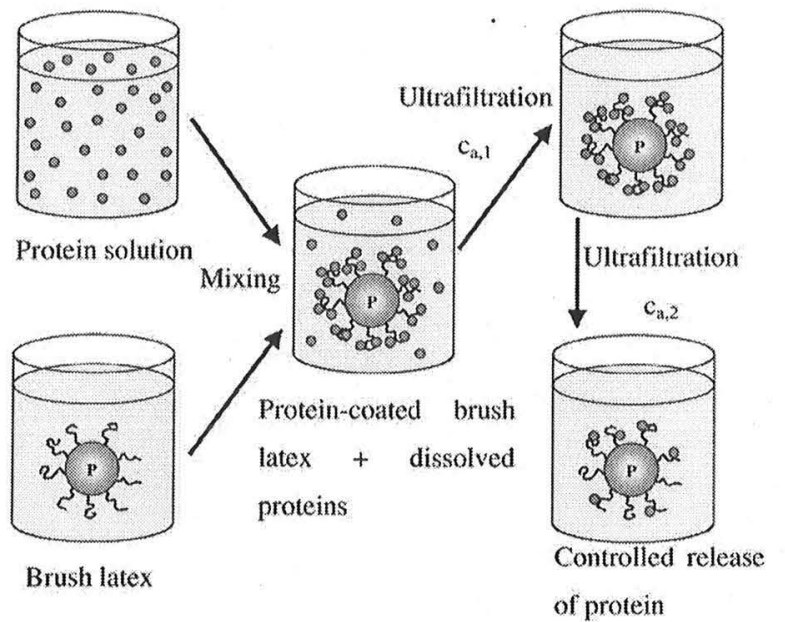

Fig. 2 Schematic representation of the PMPA: Solutions of BSA were prepared in buffer solutions with defined concentrations of added salt. These solutions were added to the SPB dissolved in the same buffer. After equilibration for $24 \mathrm{~h}$ the non-adsorbed protein was removed by careful serum replacement. ${ }^{16}$ For the desorption experiment BSA is first adsorbed onto the spherical polyelectrolyte brushes at low ionic strength $c_{a, 1}$. Unbound protein is flushed away by ultrafiltration against buffer solution of the same ionic strength. The controlled release of the BSA is induced by ultrafiltration with buffer solution of higher ionic strength $c_{\mathrm{a}, 2}{ }^{33}$

polyelectrolyte brushes (see Fig. 1) if the ionic strength is low. No adsorption takes place at high ionic strength. ${ }^{16,28,31,33}$ Fig. 2 shows this process in a schematic fashion: the protein and the spherical polyelectrolyte brush (SPB) particles are mixed in an aqueous buffer solution of defined ionic strength. ${ }^{16}$ The PMPA process takes place and the unbound protein is removed by ultrafiltration against a buffer solution of the same ionic strength. It needs to be noted that the ultrafiltration in this step is done with a large excess of solution. Yet, no protein is liberated. This experimental finding points to a non-equilibrium state within the brush layer. If there would be an equilibrium distribution of the protein between the SPB and the solution, the process of ultrafiltration would flush away all the bound protein as well. ${ }^{16}$ This, however, is not observed and the strong binding of the proteins to the SPB is to be explained by the theoretical model to be discussed in turn.

The PMPA leads to strong adsorption at low ionic strength but virtually no adsorption takes place at high salt concentrations. ${ }^{16}$ Therefore it should be possible to release protein bound at low ionic strength through raising the salt concentration again. This has been found for the fluorescent protein mEosFP indeed. ${ }^{31}$ Moreover, a recent study using BSA could demonstrate that this release proceeds in steps if the salt concentration is raised step-wise. ${ }^{33}$ This finding underscores the intimate relation between the PMPA and the electrostatic interaction between the polyelectrolyte chains and the surface of the protein.

In the subsequent section we shall delineate the main experimental findings on the PMPA process available so far. Section 4 will discuss the main driving forces for the PMPA. A brief section 5 will conclude this paper.

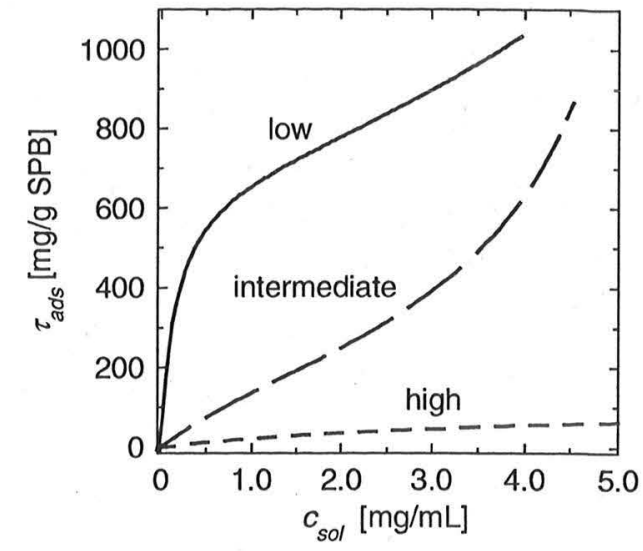

Fig. 3 Schematic representation of the adsorption of proteins onto spherical polyelectrolyte brushes. The amount of adsorbed protein per gram of the carrier particles $\tau_{\text {ads }}$ is plotted against the concentration of the protein $c_{\text {sol }}$ remaining in solution. Parameter of the curves is the concentration $c_{a}$ of added salt defining the ionic strength in the system. Strong adsorption takes place at low ionic strength whereas little protein is adsorbed if the ionic strength is high. ${ }^{16,26,32,33}$

\subsection{Polyelectrolyte-mediated protein adsorption: survey of experimental results}

The experimental findings related to the PMPA obtained so far can be summarized as follows:

(1) The decisive parameter of the PMPA is the ionic strength in the system. ${ }^{16,17,23-25,32,33}$ This is shown schematically in Fig. 3. Here the amount of adsorbed protein per gram of the SPB $\tau_{\text {ads }}$ is plotted against the concentration $c_{\text {sol }}$ of the protein remaining in solution. In this way the resulting curves resemble the usual adsorption isotherms. In some cases the adsorption is so strong that virtually no protein is left in the solution. In this case the 'isotherm' shoots up at the origin and bends over only at high adsorption degrees. ${ }^{16,26,32}$ At intermediate ionic strength the amount of protein varies linearly with $c_{\text {sol }}$ in first approximation. Only at high ionic strength does the brush layer become more and more protein-resistant. In this regime the steric repulsion between the dissolved proteins and the brush layer of the SPB finally becomes operative and only marginal adsorption results.

(2) Previous studies by FT-IR have shown that the secondary structures of the adsorbed BSA, $\beta$-lactoglobulin and ribonuclease $\mathrm{A}$ are nearly undisturbed. ${ }^{26,29}$ Moreover, the activity of adsorbed enzymes such as glucoamylase is largely preserved. ${ }^{28,30}$ The same conclusion was drawn from a study of the fluorescence activity of the fluorescent protein mEosFP. ${ }^{31}$ It thus becomes evident that the PMPA presents a very mild way of immobilizing proteins which circumvents the denaturation that may easily occur on solid surfaces. ${ }^{3}$

(3) The protein molecules are evenly distributed within the brush layer. ${ }^{27}$ This could be shown directly by a detailed analysis of the SPB before and after the adsorption of proteins by small-angle X-ray scattering (SAXS). For the details of this work the reader is referred to ref. 27 and further citations given there. Fig. 4 gives a scheme of the final result derived from SAXS which is to scale. ${ }^{27}$ The protein (BSA in this case) 


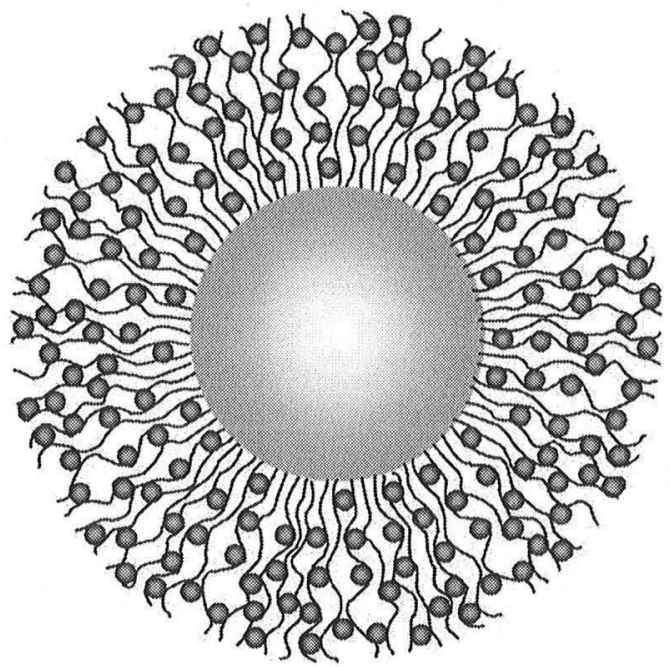

Fig. 4 Schematic representation of the protein molecules embedded into the spherical polyelectrolyte brush as derived from SAXS measurements. ${ }^{27}$ The diagram shows the dimension of a typical annealed brush system with adsorbed BSA in scale. A similar distribution within the brush layer could be derived by SAXS for RNase A. ${ }^{27}$

is closely correlated to the polyelectrolyte chains of the spherical polyelectrolyte brush. This model is qualitatively corroborated by cryogenic transmission electron microscopy ${ }^{22}$ and indicates clearly that the driving force for the PMPA must be related to the direct interaction of the proteins with the polyelectrolytes within the brush layer.

(4) As already mentioned above, most of the adsorbed proteins such as BSA can be liberated again by raising the ionic strength in the system. ${ }^{33}$ This release can be done in a controlled fashion and the amount of protein released for each raise of the ionic strength is well-defined. ${ }^{33}$

(5) The amount $\tau_{\text {ads }}$ of adsorbed protein per unit weight of the SPB is uniquely related to the concentration $c_{\text {sol }}$ of the protein remaining in the serum. It can be described by an expression which is akin the conventional BET-isotherm describing the equilibrium adsorption of multilayers. ${ }^{16,30} \mathrm{We}$ consider two fractions: one fraction of protein molecules which enters deeply into the brush layer and shows an higher adsorption energy, and a second fraction of the protein molecules which are more loosely bound in the periphery of the brush. $\tau_{\mathrm{ads}, \mathrm{M}}$ denotes the maximum mass of the strongly bound protein and $z w_{\text {ads }} c_{\text {sol }}$ with $z>1$ is the corresponding probability of the adsorption of the more strongly bound protein molecules. We assume that the adsorption energy for protein molecules located in subsequent layers is much lower and can be averaged. The probability for the adsorption for the less strongly bound protein fractions is only $w_{\text {ads }} c_{\text {sol }}$. The resulting expression reads ${ }^{30}$

$$
\frac{\tau_{\mathrm{ads}}}{\tau_{\mathrm{ads}, \mathrm{M}}}=\frac{z w_{\mathrm{ads}} c_{\mathrm{sol}}^{1 / n}}{\left(1-w_{\mathrm{ads}} c_{\mathrm{sol}}^{1 / n}\right)\left[1+(z-1) w_{\mathrm{ads}} c_{\mathrm{sol}}^{1 / n}\right]}
$$

where $n$ is an empirical parameter which can be ascribed to the dependence of the adsorption energy on the degree of adsorption. Hence, $\tau_{\text {ads }}$ as a function of $c_{\text {sol }}$ may be described in terms of four adjustable parameters $\tau_{\mathrm{ads}, \mathrm{M}}, w_{\text {ads }}, z$ and $n$. The adsorption of a number of proteins such as BSA, BLG, ribonuclease, RNase A, glucoamylase and b-glucosidase onto brushes of different spatial dimensions and either made up by weak or strong polyanions could be described by eqn (1). ${ }^{16,26,29,30}$ Hence, the expression did turn out to be of general use for the PMPA. Table 1 gathers selected results of different proteins and SPB studied quantitatively. As $\tau_{\mathrm{ads}, \mathrm{M}}$ refers to the strongly bound protein fraction, the strength of the PMPA can be thus given quantitatively in terms of $\tau_{\mathrm{ads}, \mathrm{M}}$.

\section{Polyelectrolyte-mediated protein adsorption: driving forces}

In the following we shall discuss the driving forces of the PMPA discussed so far: (1) a charge reversal of the protein by a pH lower within the brush layer than outside, ${ }^{45}$ and (2) the counterion release force. ${ }^{16,24,25,32,33,46-49}$ Both effects have already been discussed previously in a qualitative manner. ${ }^{16}$

\subsection{Charge reversal}

The localization of counterions within a weak polyelectrolyte may be followed by a smaller $\mathrm{pH}$ within the brush layer if the ionic strength is low. This effect and its implication for the PMPA was recently discussed in detail by Biesheuvel and Wittemann ${ }^{45}$ and more recently by Biesheuvel et al. ${ }^{50}$ By suitable combination of the $\mathrm{pH}$ adjusted in the system and a low ionic strength, the local $\mathrm{pH}$ within the brush layer may be lower than the pI of the protein. Hence, the net charge of the protein is reversed and a strong electrostatic attraction between unlike charged objects becomes operative. The theory of Biesheuvel and Wittemann ${ }^{45}$ therefore predicts a strong dependence on the $\mathrm{pH}$ and the adsorption is predicted to vanish for a sharply defined $\mathrm{pH}$.

Previous experiments, however, have revealed that the $\mathrm{pH}$ is a parameter of secondary importance. ${ }^{16}$ This can be seen from the discussion of Fig. 8 of ref. 16 (see also the discussion of Fig. 3): there is a very pronounced adsorption of BSA in the immediate vicinity of the isoelectric point. In this region charge reversal is certainly operative and leads to a marked adsorption of proteins. Here charge reversal is certainly the main driving force for the PMPA. However, strong adsorption still takes place at considerably higher $\mathrm{pH} .{ }^{16}$ This points to the fact that the $\mathrm{pH}$ is an important but not a decisive parameter.

\subsection{Counterion release}

The main difference between free polyelectrolyte chains as compared to a polyelectrolyte brush is the strong localization of the counterions in the latter case. Pincus ${ }^{51}$ and Borisov et al. $^{52}$ were the first to predict that most of the counterions cannot evade the brush layer but are confined within. This leads to an enormous osmotic pressure for salt-free systems ("osmotic brush") which in turn will stretch the polyelectrolyte chains of the brush layer to nearly full length. For spherical polyelectrolyte brushes it can be shown by dynamic light scattering, ${ }^{19,20}$ cryo-TEM, ${ }^{22}$ and direct osmotic measurements $^{53}$ that $95-97 \%$ of the counterions are osmotically inactive. Nearly all counterions are hence confined within 
Table 1 Modelling of the adsorption of BSA, BLG and RNase A to spherical polyelectrolyte brushes composed of either poly(acrylic acid) (SPB AB1-4) or poly(styrene sulfonic acid) (SPB QB1-2) according to eqn (1). The geometric parameters of the brushes are given as additional information: $R$ denotes the radius of the poly(styrene) core and $L$ the thickness of the brush as function of the $\mathrm{pH}$ and concentration of added salt $c_{a}$. The Donnan pressure was calculated according to eqn (2-4). See text for further explanation

\begin{tabular}{|c|c|c|c|c|c|c|c|c|c|c|c|}
\hline Protein & SPB & $\mathrm{pH}$ & $c_{a} / \mathrm{mM}$ & $R / \mathrm{nm}$ & $L / \mathrm{nm}$ & $\Pi_{\mathrm{D}} / 1000 / \mathrm{Nm}^{-2 /}$ & $\tau_{\text {ads. } \mathrm{M} / \mathrm{mg} \mathrm{g}^{-1} \mathrm{SPB}}$ & $z 1$ & $w_{\text {ads }} / \mathrm{ml} \mathrm{mg}^{-1}$ & $1 / n$ & Ref. \\
\hline BSA & $\mathrm{AB} 1$ & 6.1 & 7 & 50 & 62 & 132 & $680 \pm 40$ & $71 \pm 9$ & $0.09 \pm 0.02$ & 1 & 16 \\
\hline BSA & $\mathrm{AB} 1$ & 6.1 & 32 & 50 & 57 & 88 & $400 \pm 60$ & $40 \pm 10$ & $0.17 \pm 0.03$ & 1 & 16 \\
\hline BSA & $\mathrm{AB} 1$ & 6.1 & 57 & 50 & 53 & 72 & $250 \pm 20$ & $4 \pm 1$ & $0.161 \pm 0.004$ & 1 & 16 \\
\hline BSA & $\mathrm{AB} 1$ & 6.1 & 107 & 50 & 49 & 54 & $180 \pm 90$ & $5 \pm 1$ & $0.03 \pm 0.02$ & 1 & 16 \\
\hline BSA & $\mathrm{AB} 1$ & 6.1 & 157 & 50 & 46 & 45 & $70 \pm 20$ & $11 \pm 5$ & $0.04 \pm 0.02$ & 1 & 16 \\
\hline BSA & $\mathrm{AB} 2$ & 6.1 & 7 & 50 & 53 & 145 & $770 \pm 60$ & $280 \pm 70$ & $0.07 \pm 0.02$ & 1 & 16,26 \\
\hline BSA & AB3 & 6.1 & 7 & 50 & 93 & 37 & $1020 \pm 90$ & $260 \pm 90$ & $0.04 \pm 0.02$ & 1 & $-a$ \\
\hline BSA & AB4 & 6.1 & 7 & 50 & 12 & 935 & $390 \pm 50$ & $120 \pm 40$ & $0.05 \pm 0.03$ & 1 & $-a$ \\
\hline BSA & QB1 & 6.1 & 107 & 55 & 57 & 75 & $770 \pm 20$ & $140 \pm 20$ & $0.17 \pm 0.01$ & 1 & $-a$ \\
\hline BSA & QB1 & 6.1 & 507 & 55 & 95 & 23 & $300 \pm 10$ & $57 \pm 9$ & $0.25 \pm 0.01$ & 1 & $-a$ \\
\hline BLG & QB1 & 6.1 & 7 & 55 & 95 & 84 & $710 \pm 100$ & $(200 \pm 300)$ & $(0.001 \pm 0.002)$ & $0.6 \pm 0.5$ & 26 \\
\hline RNase A & QB2 & 9.3 & 7 & 55 & 63 & 158 & $600 \pm 30$ & $800 \pm 400$ & $0.004 \pm 0.002$ & $0.51 \pm 0.07$ & 30 \\
\hline RNase A & QB1 & 9.3 & 7 & 55 & 100 & 73 & $740 \pm 30$ & $230 \pm 60$ & $0.008 \pm 0.002$ & $0.53 \pm 0.04$ & 26,30 \\
\hline
\end{tabular}

the brush layer. The high osmotic pressure thus created determines the structure and interaction of these particles. ${ }^{21}$ This is quite the opposite to what is found for linear polyelectrolytes in solution. Here only the Manning fraction of the counterions is immobilized by the electric field of the linear macroion. ${ }^{54,55}$ For typical polyelectrolytes such as poly(acrylic acid) used for the synthesis of the SPB, this fraction is of the order of $75 \%$, that is, the osmotic coefficient giving the fraction of free counterions is around 0.25 .

The driving force for the PMPA can now be discussed in terms of the number of released counterions. ${ }^{16,32,33}$ We consider the uptake of a protein from solution as shown in Fig. 5. Here we enumerate the net release of counterions in this process. Because $\mathrm{pH}>\mathrm{pI}$, the number $N_{-}$of negatively charged groups on its surface is slightly greater than $N_{+}$, the number of positively charged groups on the surface. Evidently, these charges must be balanced by an equally high number of counterions of the opposite sign. Therefore each protein carries along $N_{+}$negative and $N_{-}$positive counterions. We
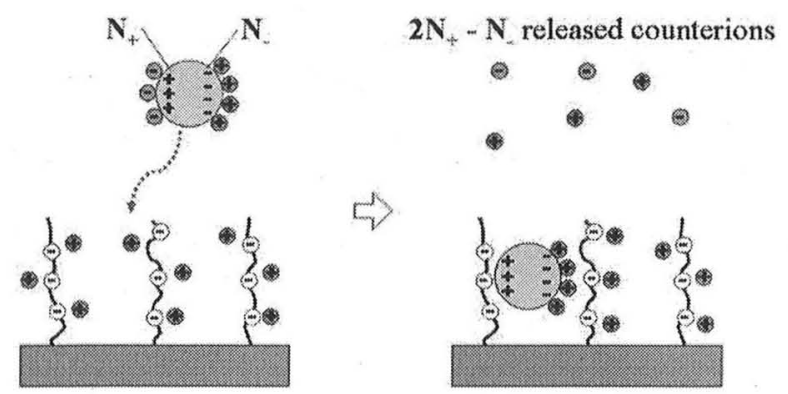

Fig. 5 Enumeration of the released counter- and coions during the process of protein adsorption. $N_{-}$is the number of negatively charged groups on the surface of the protein which is slightly greater than $N_{+}$, the number of positively charged groups on the surface $(\mathrm{pH}>\mathrm{pI})$. For each protein molecule a total of $\Delta N=2 N_{+}-N_{-}>0$ counterions of the protein and the brush layer is released. The decrease of the Donnan pressure $\Pi_{\mathrm{D}}(c f$. eqn (4)) within the brush layer thus obtained leads to the strong adsorption of proteins at low ionic strength. At high salt concentration $c_{a}$ the effect must vanish because $\Pi_{\mathrm{D}}=0$ in this case. See text for further explanation. ${ }^{32,33}$ now consider the immersion of the protein in the brush layer (see Fig. 5). The $N_{+}$positive charges on the surface of the protein now become counterions of the negatively charged polyelectrolyte chains. As a consequence, $N_{+}$positive counterions previously immobilized within the brush layer as well as the $N_{+}$negative counterions formerly carried along by the protein in solution are released. On the other hand, the $N_{-}$ negative charges on the surface of the protein carry along their $N_{-}$positive counterions which will increase the number of small ions within the brush layer again. The balance between the release and the uptake, however, is positive since a total of $\Delta N=2 N_{+}-N_{-}>0$ counterions have been released in this process. The concomitant lowering of the osmotic pressure within the brush layer is of the order of $k T \Delta N$ divided by the volume of the brush (see Fig. 1).

A point to be made in conjunction with Fig. 5 is the size of the patches on the surface. These patches must necessarily be of a minimum size to ensure the strong correlation of the respective counterions. A single charge on the surface would not localize the counterion and there would be no difference upon putting the protein into the brush layer. However, patches of a few charges will bind their counterions more strongly and become multivalent counterions in turn when the protein is located within the brush layer. Therefore the counterion release mechanism is bound to the presence of charged patches on the surface of the proteins. Hence, the above balancing leading to $\Delta N$ must be restricted to the number of localized counterions.

\subsection{Counterion release: a simple model}

The foregoing considerations can be put into more quantitative terms as follows: we suggest here that the strong osmotic pressure inside the brush layer is one of the driving forces for the PMPA. This can be argued in the following way: nearly all of the counterions are confined within the brush layer and the osmotic pressure within the brush layer can be treated in terms of the classical Donnan equilibrium. This approach has already been suggested by Russel and coworkers ${ }^{56}$ and applied successfully to the systems under consideration here. ${ }^{19,20,32,33}$ 
The brush layer is characterized by its thickness $L$ ( $c f$. Fig. 1). The concentration of the counterions $c_{\mathrm{ci}}$ is given by the number of charges within the brush layer because we assume full dissociation. However, following Russel and coworkers, ${ }^{56}$ counterion condensation is taken into account by assuming that counterions will condense onto the polyelectrolyte chains until the distance between two charges is given by the Bjerrum length $l_{\mathrm{B}}\left(l_{\mathrm{B}}=0.714 \mathrm{~nm}\right.$ in water at $\left.25^{\circ} \mathrm{C}\right)$. Therefore $c_{\mathrm{ci}}$ is given $b y^{56}$

$$
c_{\mathrm{ci}}=\frac{3 R^{2} \sigma L_{\mathrm{c}}}{l_{\mathrm{B}}\left[(R+L)^{3}-R^{3}\right] N_{\mathrm{L}}}
$$

where $R$ is the radius of the core particles, $\sigma$ is number of grafted chains per $\mathrm{nm}^{2}$, and $L_{\mathrm{c}}$ is the contour length of the grafted chains ( $c f$. Fig. 1). Given the concentration $c_{a}$ of added salt, the total concentration $c_{c}$ of all ionic species within the brush layer follows from the Donnan equilibrium as

$$
c_{c}=2 c_{a}\left[\left(\frac{c_{\mathrm{ci}}}{2 c_{a}}\right)^{2}+1\right]^{1 / 2}
$$

From this the Donnan pressure $\Pi_{\mathrm{D}}$ within the brush layer is given by

$$
\Pi_{\mathrm{D}}=R T\left(c_{c}-2 c_{a}\right)
$$

Note that $\Pi_{\mathrm{D}}$ is fully given by known experimental parameters. In particular, the thickness $L$ of the brush layer can be determined by dynamic light scattering with great precision.

We now may correlate the parameter $\tau_{\text {ads,M }}$ (see eqn (1)) with the Donnan pressure $\Pi_{\mathrm{D}}$ within the brush layer. The parameter $\tau_{\text {ads, } M}$ gives a quantitative measure for the amount of strongly bound protein and can be compared for different systems. For a quantitative comparison, however, one has to keep in mind that the thickness $L$ depends on $c_{a}$ as well as on the system, that is, on the contour length $L_{\mathrm{c}}$. To ensure a meaningful comparison, $\tau_{\mathrm{ads}, \mathrm{M}}$, is normalized to the volume $V_{\mathrm{s}}$ of the brush layer given by

$$
V_{\mathrm{S}}=\frac{4 \pi}{3}\left[(R+L)^{3}-R^{3}\right]
$$

Fig. 6 displays the comparison between $\tau_{\text {ads, } M} / V_{s}$ and $\Pi_{\mathrm{D}}$. The respective data have been taken from Table 1. Evidently, there is a correlation of the concentration of $\tau_{\mathrm{ads}, \mathrm{M}} / V_{\mathrm{s}}$ with $\Pi_{\mathrm{D}}$ for at least three proteins and brush particles composed of either weak or strong polyelectrolytes and different geometric parameters. The four data points in Fig. 1 which are located directly on the dashed line all refer to the adsorption of BSA on the same brush system (ABI in Table 1) but at different ionic strength $c_{a}$.

The model as discussed in conjuction with Fig. 6 has a certain predictive power: $\tau_{\mathrm{ads}, \mathrm{M}} / V_{\mathrm{s}}$, that is, the PMPA will become stronger with a higher Donnan pressure and vanish concomitantly at sufficiently high salt concentration within the system. Moreover, planar brushes that have a higher average concentration of the polyelectrolyte chains within $V_{\mathrm{s}}$ will exhibit a higher adsorption. It should be noted that this limit is approached by system AB4 of Table 1. Here the respective $\tau_{\text {ads, } M} / V_{\mathrm{s}}$ lies directly on the line defined by linear regression through all the data points. Hence, the Donnan pressure and

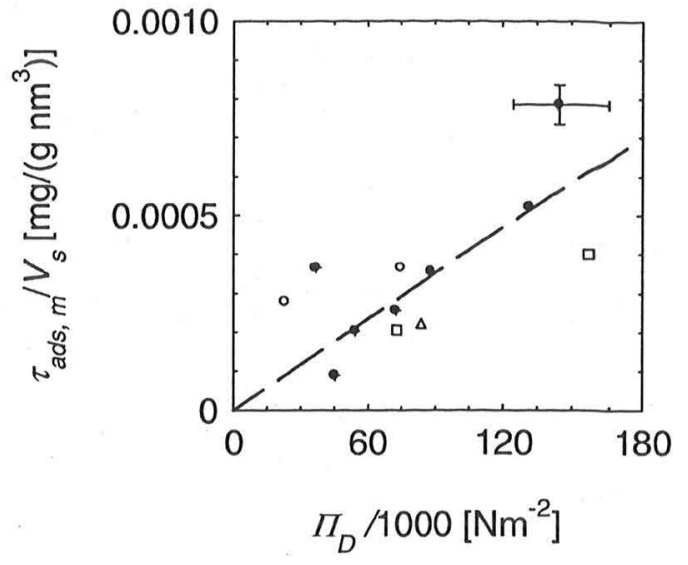

Fig. 6 Modelling the dependence of the PMPA on ionic strength in the solution: The amount of strongly adsorbed protein $\tau_{\mathrm{ads}, \mathrm{M}}$ (see eqn (1)) per volume $V_{\mathrm{s}}$ (eqn (5)) of the brush layer is plotted against the Donnan pressure $\Pi_{\mathrm{D}}$ in the brush layer (eqn (4). Closed circles: Adsorption of BSA onto brushes made up by of poly(acrylic acid) (systems AB1-3 in Table 1). ${ }^{16,26}$ Open circles: Adsorption of BSA onto brushes made up by poly(styrene sulfonic acid) (systems QB1-2 in Table 1). Open squares: Adsorption of ribonuclease $A$ onto brushes made up by poly(styrene sulfonic acid) (systems QB1-2 in Table 1). ${ }^{26.29}$ Open triangle: Adsorption of BLG onto brush QB1 made up by poly(styrene sulfonic acid). ${ }^{26}$ The dashed line displays the linear regression through all the points. The experimental data is taken from Table 1. See text for further explanation.

the uptake of the protein molecules into the brush layer are closely related to each other. This has to indeed be the case if the protein adsorption is related to the release of counterions from the brush layer and therefore to a lowering of the osmotic pressure within the brush as shown in section 3.2. In other words, the Donnan pressure seems to be a characteristic measure for the PMPA and can thus be used to predict the degree of adsorption of a given protein from the geometric parameters of the polyelectrolyte brush at a given salt concentration $c_{a}$. Further investigations are under way for testing this prediction in more detail.

\section{Conclusions}

Concluding this review one can state that the PMPA and the interaction of proteins with free polyelectrolyte chains seem to be closely related phenomena: both can take place on the "wrong side" of the isoelectric point and the ionic strength within the system is one of the decisive factors. Moreover, both the PMPA and the interactions with linear polyelectrolytes can be traced back to the presence of positive patches. These patches have to be of a minimum size to ensure strongly correlated counterions. A simple consideration of the release and uptake of counterions did show that the PMPA leads to a net release of numerous counterions and presents a strong driving force for the PMPA. As the correlation of the counterions within the brush layer is much stronger than the one with linear polyelectrolytes, it is obvious that counterion release forces lead to much stronger protein binding. 
The PMPA makes the SPB protein carriers with many possible uses. Protein molecules can be reversibly uptaken at low ionic strength and released by raising the amount of added salt. Moreover, the structural integrity of the native protein remains unaltered during the PMPA. Thus, the activity of immobilized enzymes is retained. The PMPA especially in combination with SPB may be of general use in terms of multiple technical applications in biotechnology as carrier of enzymes. ${ }^{1-4,29,30}$

\section{References}

1 Physical Chemistry of Biological Interface, ed. A. Baskin and W. Norde, Marcel Dekker, New York, 1999.

2 W. Senaratne, L. Andruzzi and Ch. Ober, Biomacromolecules, 2005, 6, 2427

3 C. Czeslik, Z. Phys. Chem., 2004, 218, 771.

4 H. Kawaguchi, Prog. Polym. Sci., 2000, 25, 1171.

5 C. F. Wertz and M. M. Santore, Langmuir, 2001, 17, 3006.

6 C. F. Wertz and M. M. Santore, Langmuir, 2002, 18, 706.

7 C. F. Wertz and M. M. Santore, Langmuir, 2002, 18, 1190.

8 C. Czeslik and R. Winter, Phys. Chem. Chem. Phys., 2001, 3, 235.

9 G. Jackler, R. Steitz and C. Czeslik, Langmuir, 2002, 18, 6565.

10 C. Czeslik, C. Royer, T. Hazlett and W. Mantulin, Biophys. J., 2003, 84, 2533.

11 G. Fragneto, T. J. Su, J. R. Lu, R. K. Thomas and A. R. Rennie, Phys. Chem. Chem. Phys., 2000, 2, 5214

12 W. Norde and J. Lyklema, J. Colloid Interface Sci., 1978, 66, 266.

13 J. H. Lee, H. B. Lee and J. H. Andrade, Prog. Polym. Sci., 1995, 20, 1043.

14 E. P. K. Currie, J. Van der Gucht, O. V. Borisov and M. A. Cohen Stuart, Pure Appl. Chem., 1999, 71, 1227, and further citations given there.

15 M. Zolk, F. Eisert, J. Pipper, S. Herrwerth, W. Eck, M. Buck and M. Grunze, Langmuir, 2000, 16, 5849.

16 A. Wittemann, B. Haupt and M. Ballauff, Phys. Chem. Chem. Phys., 2003, 5, 1671.

17 G. Jackler, A. Wittemann, M. Ballauff and C. Czeslik, Spectroscopy, 2004, 18, 1671.

18 X. Guo, A. Weiss and M. Ballauff, Macromolecules, 1999, 32, 6043.

19 X. Guo and M. Ballauff, Langmuir, 2000, 16, 8719.

20 X. Guo and M. Ballauff, Phys. Rev. E, 2001, 64, 051406

21 A. Jusufi, C. N. Likos and M. Ballauff, Colloid Polym. Sci., 2004, 282, 910 .

22 A. Wittemann, M. Drechsler, Y. Talmon and M. Ballauff, J. Am, Chem. Soc., 2005, 127, 9688.

23 C. Czeslik, G. Jackler, T. Hazlett, E. Gratton, R. Steitz, A. Wittemann and M. Ballauff, Phys. Chem. Chem. Phys., 2004, 6, 5557.

24 C. Czeslik, G. Jackler, R. Steitz and H.-H. V. Grünberg, J. Phys. Chem. B, 2004, 108, 133956.

25 O. Hollmann and C. Czeslik, Langmuir, 2006, 22, 3300.

26 A. Wittemann and M. Ballauff, Anal. Chem., 2004, 76, 2813.
27 S. Rosenfeldt, A. Wittemann, M. Ballauff, E. Breininger, J. Bolze and N. Dingenouts, Phys. Rev. E, 2004, 70, 016403

28 Th. Neumann, B. Haupt and M. Ballauff, Macromol. Biosci., 2004 4,13

29 A. Wittemann and Ballauff, Macromol. Biosci., 2005, 5, 13.

30 B. Haupt, Th. Neumann, A. Wittemann and M. Ballauff, Biomacromolecules, 2005, 6, 948.

31 K. Anikin, C. Röcker, A. Wittemann, J. Wiedenmann, M. Ballauff and G. U. Nienhaus, J. Phys. Chem. B, 2005, 109, 5418.

32 A. Wittemann, B. Haupt and M. Ballauff, Prog. Colloid Polym. Sci., 2006, 133, 58.

33 A. Wittemann, B. Haupt and M. Ballauff, Z. Phys. Chem., NF, in press.

34 C. L. Cooper, P. L. Dubin, A. B. Kayitmazer and S. Turksen, Curr. Opin. Colloid Interface Sci., 2005, 10, 52.

35 C. G. de Kruif, F. Weinbreck and R. de Vries, Curr. Opin. Colloid Interface Sci., 2004, 9, 340.

36 Proteins, Amino Acids and Peptides, ed. E. J. Cohn and J. T. Edsall, Reinhold Publishing Corporation, New York, 1943.

37 Ch. Tanford, Physical Chemistry of Macromolecules, John Wiley, New York, 1961.

38 H. G. Bungenberg de Jong and H. R. Kruyt, Proc. K. Ned. Akad. Wet., 1929, 32, 849 .

39 H. G. Bungenberg de Jong, in Crystallization, coacervation, flocculation, ed. H. R. Kruyt, Colloid Science, Elsevier Publishing, Amsterdam, 1949, vol. II, ch. VIII, p. 232.

40 K. R. Grymonpre, B. A. Staggemeier, P. L. Dubin and K. W. Mattison, Biomacromolecules, 2001, 2, 422.

41 E. Seyrek, P. L. Dubin, Ch. Tribet and E. A. Gamble, Biomacromolecules, 2003, 4, 273.

42 T. Hattori, R. Hallberg and P. L. Dubin, Langmuir, 2000, 16, 9738.

43 W. A. Bowman, M. Rubinstein and J. S. Tan, Macromolecules, 1997, 30, 3262.

44 R. de Vries, F. Weinbreck and C. G. de Kruif, J. Chem. Phys., 2003, 118, 4649.

45 P. M. Biesheuvel and A. Wittemann, J. Phys. Chem. B, 2005, 109, 4209.

46 M. T. Record, C. F. Anderson and T. M. Lohman, Q. Rev. Biophys., 1978, 11, 103.

47 P. Sens and J.-F. Joanny, Phys. Rev. Lett., 2000, 84, 4862.

48 C. Fleck and H. H. von Grünberg, Phys. Rev. E, 2002, 63, 061804.

49 A. A. Meier-Koll, C. C. Fleck and H. H. von Grünberg, J. Phys.: Condens. Matter, 2004, 16, 6041

50 P. M. Biesheuvel, F. A. M. Leermakers and M. A. Cohen Stuart, Phys. Rev. E, 2006, 73, 011802.

51 P. Pincus, Macromolecules, 1991, 24, 2912

52 O. V. Borisov, T. M. Birshtein and E. B. Zhulina, J. Phys. II, 1991, 1, 521 .

53 B. Das, X. Guo and M. Ballauff, Prog. Colloid Polym. Sci., 2002, $121,34$.

54 G. Manning, Annu. Rev. Phys. Chem., 1972, 23, 117, and further references cited therein.

55 See the discussion of this point in M. Deserno, Ch. Holm, J. Blaul, M. Ballauff and M. Rehahn, Eur. Phys. J. E, 2001, 5, 97.

56 R. Hariharan, C. Biver and W. B. Russel, Macromolecules, 1998, $31,7514$. 